\section{COMMENT}

The details of C.P.K. activity are shown in Fig. 1, 2, and 3. The serum lithium levels for each patient are within acceptable limits (Fry and Marks, 1971).

Six male and nine female patients under the continuous care of other clinicians were also found to have large increases in C.P.K. activity at a time when they were clinically well and had acceptable serum lithium levels. We received blood at intervals varying from two weeks to two months for serum lithium estimation from 140 patients, 91 of whom were female. In all cases when seen at subsequent interviews the patients were found to have been clinically well at the time of raised C.P.K. activity.

\section{Discussion}

Large increases in C.P.K. activity have been shown to occur in many patients suffering from psychotic illness (Meltzer et al., 1969; Gosling et al., 1972). The raised activity of serum C.P.K. lasts for three to four days after an acute episode of cell necrosis -for example, myocardial infarction (Warburton, Bernstein, and Wright, 1965). The increase in C.P.K. occurs early in the psychotic episode and its median duration is 10 days (Meltzer et al., 1969). This is consistent with continued release of the enzyme into the serum. The increase in serum C.P.K. can precede the psychological manifestations of the illness by a few days (Fig. $1 \mathrm{X}$, Fig. $2 \mathrm{X}$ ). For these reasons the times of increased C.P.K. activity are easily missed in patients who may attend an outpatient clinic at infrequent intervals. We regard a raised serum C.P.K. (in the absence of physical illness) as indicative of either an impending or an established psychotic episode (Gosling et al., 1972).

Long symptom-free periods while taking lithium carbonate may be due either to a long period of remission or to the prophylactic action of the lithium ion. The routine estimation of C.P.K. levels at the time of lithium estimation has shown that lithium does not prevent the increased C.P.K. activity in those patients who have been shown clinically to be lithium responders. These raised levels of C.P.K. activity in patients responding to prophylactic lithium suggest that the basic mechanism of the illness is still present despite the clinically normal mood. In Cases 1 and 2 it is seen that before starting lithium carbonate psychotic illness requiring admission to hospital was always associated with raised C.P.K. activity. Similar increases in C.P.K. activity since starting lithium were not accompanied by mood changes and these might have corresponded to times when the patients would have had psychotic illnesses. The other patient (Case 3), who had been well on prophylactic lithium for eight years, still has peaks of C.P.K. activity, probably indicating the continued need to take lithium carbonate. It will be noted that in these three patients the C.P.K. level, while on lithium, was always at or slightly above the upper limit of normal. We are unable to account for this phenomenon since it has not been observed in the other patients attending the lithium clinic.

Changes in C.P.K. patterns are seen in the sera referred to this laboratory by other clinicians who have reported that the patients are clinically well on lithium therapy. These findings are in agreement with the observations on our own patients.

It is concluded that lithium carbonate controls or prevents manic depressive illness even when the disease remains active as shown by the recurring peaks of raised C.P.K. activity. They also show that lithium carbonate must be continued for long periods as one patient at least is still at risk after being on lithium carbonate for eight years.

We wish to thank Mr. E. Foster and Mrs. J. White for preparing the graphs and Dr. A. J. N. Warrack for helpful criticism.

\section{References}

Blackwell, B., and Shepherd, M. (1968). Lancet, 1, 968.

Fry, D. E., and Marks, V. (1971). Lancet, 1, 886.

Gosling, R., Kerry, R. J., Orme, J. E., and Owen, G. (1972). British fournal

of Psychiatry. In press.
Meltzer, H., Elkun, L., and Moline, R. A. (1969). Archives of General Psychiatry, 21, 731.

Warburton, F. G., Bernstein, A., and Wright, A. C. (1965). British Heart fournal, 27, 740 .

\title{
Chloramphenicol Resistance in the Typhoid Bacillus
}

\author{
E. S. ANDERSON, H. R. SMITH
}

British Medical Fournal, 1972, 3, 329-331

\section{Summary}

Chloramphenicol resistance has been reported in individual strains of Salmonella typhi since 1950, but there had been no accounts of epidemics caused by resistant strains of the organism until 1972, when one occurred in Mexico. Two British patients have been infected in that country, and the organism isolated from them corresponds in all respects with the description of the Mexican epidemic strain of $S$. typhi: it is a degraded Vi strain resistant to chloramphenicol, streptomycin, sulphonamides, and tetracyclines. It owes its resistance to an $R$ factor which can be transferred to Escherichia coli and thence to drugsensitive $S$. typhi. Although there is a negligible risk of \footnotetext{
Enteric Reference Laboratory, Public Health Laboratory Service,
London N.W.9

E. S. ANDERSON, M.D., F.R.C.PATH., F.R.S., Director

H. R. SMITH, M.A., Member of Scientific Staff
}

\begin{abstract}
dissemination of this strain in Britain the incident is a reminder of the need to restrict the use of drugs such as chloramphenicol to the serious diseases for which they are virtually specific.
\end{abstract}

\section{Introduction}

Chloramphenicol resistance in Salmonella typhi was apparently first reported in England (Colquhoun and Weetch, 1950). It was subsequently observed in India (Murti et al., 1962), West Africa (Njoku-Obi and Njoku-Obi, 1965), and Greece and Israel (Kontomichalou, 1967; Sompolinsky et al., 1967). The resistance in the last two instances was caused by a transferable extrachromosomal element known as a resistance factor or $\mathbf{R}$ factor.

\section{Studies at Enteric Reference Laboratory}

The minimal inhibitory concentration (M.I.C.) of chloramphenicol for drug-sensitive $S$. typhi is between 1 and $3 \mu \mathrm{g} / \mathrm{ml}$ with the technique routinely used in the Enteric Reference Labora- 
tory. We have examined seven chloramphenicol-resistant strains of $S$. typhi received between 1966 and 1969. All were foreign in origin. Two were sent from Chile in 1966. One of these belonged to Vi phage type 46 and had a chloramphenicol M.I.C. of $150 \mu \mathrm{g} / \mathrm{ml}$. The other Chilean culture belonged to Vi phage type A, and its chloramphenicol M.I.C. was $12.5 \mu \mathrm{g} / \mathrm{ml}$. A strain of $S$. typhi was isolated in 1967 in Norwich from a patient infected in Aden. This was a degraded Vi strain with a chloramphenicol M.I.C. of about $6.0 \mu \mathrm{g} / \mathrm{ml}$.

These three strains were resistant to chloramphenicol only, and we were unable either to transfer the resistance to recipient strains or to mobilize it with a transfer factor-the agent responsible for the transferability of $R$ factors (Watanabe, 1963; Anderson, 1965a, 1965b; Anderson, 1966).

Three further chloramphenicol-resistant $S$. typhi strains, two belonging to Vi phage type $A$ and one to Vi phage type E1, were received in 1967 from Kuwait. All were resistant to ampicillin (A), chloramphenicol (C), and tetracyclines (T). The M.I.C.s of the respective drugs for the parent strains were: ampicillin $250 \mu \mathrm{g} / \mathrm{ml}$, chloramphenicol $250 \mu \mathrm{g} / \mathrm{ml}$, and tetracyclines $62.5 \mu \mathrm{g} / \mathrm{ml}$. The resistances were transferred en bloc to Escherichia coli $\mathrm{K} 12$ at high frequency (greater than $50 \%$ ) in overnight crosses. The resulting lines transferred the ACT resistance factor at a frequency of about $3 \times 10^{-3}$ to the type strains of Vi type A and E1, of $S$. typhi, which are used as standard recipients in the study of $R$ factors (Anderson, 1966, 1968).

The ACT factor in the Kuwait strains appeared to be uniform throughout. This was interesting, because each patient was suspected to have been infected in a different place-one in Aden, one in Cairo, and one in Pakistan. Two of the strains were isolated by blood culture and one from stools. It can be concluded either that this ACT resistance factor is widely distributed in the Middle East and Pakistan or that the infecting $S$. typhi strains acquired it in Kuwait. The latter explanation seems the more probable, since we have no evidence from strains of $S$. typhi sent from the areas other than Kuwait that the ACT factor is common elsewhere.

According to current classification of $\mathrm{R}$ factors the ACT factor is categorized as fertility inhibition $+\left(f^{+}\right)$and F-like (Meynell et al., 1968). It probably originated in a non-pathogenic intestinal commensal such as $E$. coli, and may well have been common in the Kuwait area during the period in which the three resistant $S$. typhi strains were encountered. The occurrence of the ACT resistance factor in $S$. typhi may thus be a reflection of the epidemiology of the $R$ factor itself, as distinct from that of its pathogenic host.

In September 1969 we received a strain of type C1 S. typhi isolated in London by blood culture from a young man probably infected in Spain. This strain was resistant to chloramphenicol, streptomycin (S), and sulphonamides (Su). The resistances were transferred en bloc at low frequency (about $10^{-4}$ ) into $E$. coli $\mathrm{K} 12$, and thence at only about $10^{-6}$ into Vi types $A$ and C1 S. typhi in overnight crosses. Despite its low transfer frequency the CSSu resistance factor is very stable, both in its original $S$. typhi host and in the type strains of $\mathrm{A}$ and $\mathrm{Cl}$ to which it was transferred. This $\mathbf{R}$ factor is of special interest because it is $f^{-}$and belongs to a new group of enterobacterial plasmids which we have designated "group H" (N. D. F. Grindley, J. N. Grindley, and E. S. Anderson, in preparation). The chloramphenicol M.I.C. of the original host strain is about $150 \mu \mathrm{g} / \mathrm{ml}$.

\section{Chloramphenicol-resistant S. typhi from Mexico}

The incidents described above appeared to be discrete, and hitherto we had no indication that they represented the acquisition of chloramphenicol resistance by $S$. typhi with sufficient frequency for epidemiologically important resistant strains of the pathogen to emerge. Recently, however, the Center for Disease Control (C.D.C.) in Atlanta, Georgia, U.S.A., reported a widespread and protracted outbreak of typhoid fever in Mexico, which started early in 1972 (C.D.C. Weekly Report, 1972a). The strain of $S$. typhi concerned carries a transferable factor with the resistance pattern CSSuT. Two American tourists have been infected with this strain (C.D.C. Weekly Report, 1972b).

Two British men who recently visited Mexico independently have developed typhoid fever. The cultures of $S$. typhi isolated in England from these patients correspond precisely with the C.D.C. description of the Mexican strain; they belong to a degraded Vi strain and they carry a CSSuT resistance factor. The M.I.C. of chloramphenicol for these Mexican strains is about $150 \mu \mathrm{g} / \mathrm{ml}$.

This $\mathrm{R}$ factor transfers from the resistant $S$. typhi to $E$. coli $\mathrm{K} 12$ at a frequency of about $10^{-4}$, and from $E$. coli $\mathrm{K} 12$ to $\mathrm{Vi}$ type A $S$. typhi at about $10^{-6}$ in overnight crosses. Like the CSSu factor described above, which came from Spain, it is $\mathrm{fi}^{-}$and belongs to group $\mathrm{H}$. However, apart from the presence of tetracycline resistance it can be distinguished from the Spanish $R$ factor by phage restriction techniques (Anderson, 1966, 1968).

\section{Discussion}

It can be assumed that chloramphenicol resistance will appear in $S$. typhi from time to time as the result of either mutation of the acquisition of $\mathbf{R}$ factors, but the Mexican outbreak seems to be the first example of its appearance in an epidemic strain. This is the type of event most feared by those who are studying the ecology of $\mathbf{R}$ factors, because chloramphenicol is the drug of choice in the treatment of typhoid fever. As the C.D.C. points out, it may not be coincidental that an $\mathbf{R}$ factor carrying the same resistances is present in a strain of Shigella dysenteriae 1 which has afflicted central America since 1968, causing severe dysentery with a high mortality. It remains to be seen whether the CCSuT resistance factor now in the epidemic typhoid strain is the same as that in the dysentery strain. If so, it could have entered the typhoid bacillus either direct as the result of contact between the dysentery and typhoid strains or by way of an intestinal commensal such as $E$. coli, which could act as a vector of the $\mathbf{R}$ factor. In any event it can be accepted that the $\mathrm{R}$ factor in this strain of $S$. typhi originated, however remotely, in one of the non-pathogenic enterobacteria.

The ultimate appearance of epidemic strains of $S$. typhi carrying $\mathbf{R}$ factors coding for chloramphenicol resistance is most likely in countries where two conditions are satisfied. The first is that typhoid fever must be common, so that the organism is frequently present in the human intestine. The second condition is that chloramphenicol should be used indiscriminately, so that its widespread selective pressure will promote the emergence of stable $\mathbf{R}$ factors coding for the respective resistance. Both these conditions are satisfied in Mexico; it is a country with a relatively high incidence of typhoid fever, and not only is chloramphenicol used on a large scale by doctors but it can be bought by the general public.

The C.D.C. commented that "although detailed studies have not been completed, it appears that many of the patients infected with resistant strains responded poorly to therapy with chloramphenicol, necessitating the use of other antibiotics, principally ampicillin" (C.D.C. Weekly Report, 1972a).

The poor response to chloramphenicol is hardly surprising, but ampicillin, to which the Mexican strain is sensitive in vitro, has proved a disappointment in general for the treatment of typhoid fever, and it would be an error to regard it as anything better than a second-line drug in this respect.

The appearance of this strain of $S$. typhi in British patients is yet another demonstration that with the extent and speed of modern travel infection can be rapidly conveyed between widely separated countries. If the causal organisms have additional unwelcome features such as chloramphenicol resistance in $S$. typhi, they present the patients concerned with 
an augmented risk, because the most effective therapy cannot be used.

Although the importation of chloramphenicol-resistant $S$. typhi is undesirable it need not arouse alarm in Britain, because opportunities for the spread of typhoid in this country are negligible. Nevertheless, if epidemics caused by chloramphenicol-resistant $S$. typhi occur on a sufficiently large scale in countries of high typhoid incidence, such organisms may spread to other countries often enough to present an irksome problem, and the possibility of residual carriers of these imported strains cannot be discounted. The British cases of typhoid infected in Mexico, and the epidemic which caused them, are a warning of this, and are a reminder that if antibiotics such as chloramphenicol are to retain their efficacy for important diseases, their use should be largely if not entirely restricted to those diseases throughout the world.

\section{References}

Anderson, E. S. (1965a). British Medical fournal, 2, 1289.

Anderson, E. S. (1965b). Nature, 208, 1016.

Anderson, E. S. (1966). Nature, 209, 637.

Anderson, E. S. (1968). Annual Review of Microbiology, 22, 131.

Center for Disease Control Weekly Report (1972a). Morbidity and Mortality, 21, 177.

Center for Disease Control Weekly Report (1972b). Morbidity and Mortality, 21, 193.

Colquhoun, J., and Weetch, R. S. (1950). Lancet, 2, 621.

Colquhoun, J., and Weetch, R. S. (1950). Lancet, 2, 621. Meynell, E., Meynell, G. G., and Datta, N. (1968). Bacteriological Reviews,

Murti, B. R., Rajyalakshmi, K., and Bhaskaran, C. S. (1962). Journal of Clinical Pathology, 15, 544 . Njoku-Obi, A. N., and Njoku-Obi, J. C. (1965). Fournal of Bacteriology,

Sompolinsky, D., Ben-Yakov, M., Aboud, M., and Boldur, I. (1967). Mutation Research, 4, 119

Watanabe, T. (1963). Bacteriological Revierws, 27, 87.

\section{MEDICAL MEMORANDA}

\section{Tuberculous Ulcer of the Skin}

\author{
HAMID SAHEBJAMI, DONALD MASSARO
}

British Medical fournal, 1972, 3, 331-332

The presence of a pulmonary infiltrate and an ulcerating chest wall lesion usually leads one to consider the diagnosis of actinomycosis. Tuberculous skin lesions, especially skin ulcers, are extremely unusual (Duncan, 1968; Olurin and Itayemi, 1970). When present they are usually ascribed to haematogenous dissemination from a pulmonary or extrapulmonary focus (Kleid and Rosenburg, 1970). We report the present case because of the rarity of tuberculous skin ulcers and because it suggests the possibility of contiguous spread of pleuropulmonary tuberculosis to the skin.

\section{Case Report}

A 58-year-old labourer was admitted to the Veterans Administration Hospital, Washington, complaining of a productive cough and weight loss for one year. Several months before admission he noted a "lump" in his right lateral chest wall which gradually enlarged and ulcerated and drained blood-tinged purulent material. He did not recall any trauma to this area. He had been exposed to heavy cement dust for 15 years and had smoked one packet of cigarettes a day for 40 years.

Physical examination showed nothing abnormal except for rales over both lungs and a round pinkish grey ulcer over the right midaxillary line at the fourth intercostal space. The ulcer was deep and indurated with raised edges and had vegetations and crusts (Fig. 1).

A chest roentgenogram showed bilateral infiltration and cavitation and pleural thickening over the right lateral chest. His intermediatestrength purified protein derivative was positive and his sputum contained acid-fast bacilli on smear and grew Mycobacterum tuberculosis on culture. Biopsy of the chest wall ulcer showed a caseating granulomatous abscess (Fig. 2). Special tissue stains did not show acid-fast bacilli or fungi. Serological studies and sputum cultures for fungi were negative.

Pulmonary Division, Veterans Administration Hospital, George Washington University Medical Center, Washington D.C. 20422 U.S.A. HAMID SAHEBJAMI, M.D.

DONALD MASSARO, M.D., Professor of Medicine

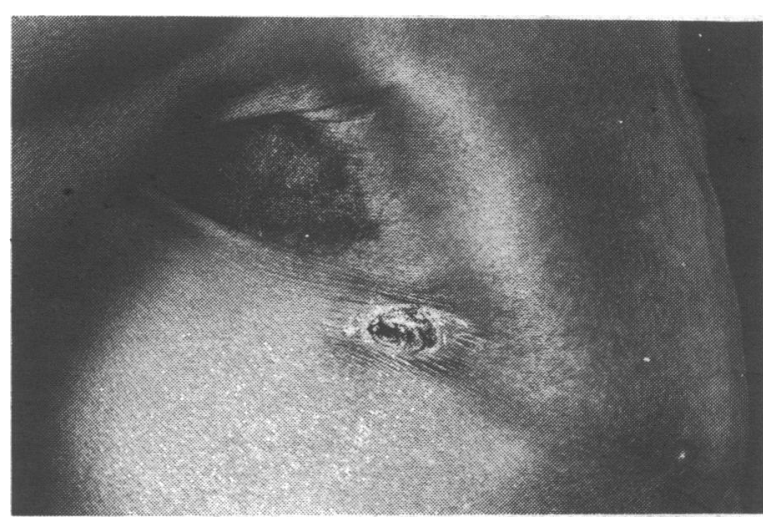

FIG. I Skin ulcer over right midaxillary line before therapy.

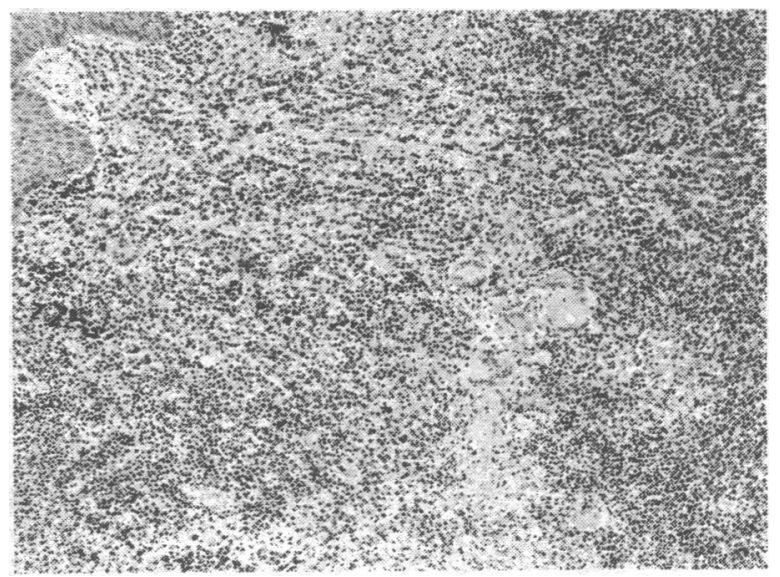

FIG. 2-Biopsy specimen of chest wall ulcer showing caseating granulomatous abscess. ( $x$ 76.)

He was treated with isoniazid, para-aminosalicylic acid, and streptomycin. The skin ulcer healed after six weeks of therapy and his sputum became negative for $M$. tuberculosis after two months of therapy.

\section{Comment}

The major purpose of this report is simply to call attention to the preserce of tuberculous skin lesions. In the present 